\title{
Thermotherapy of Buruli Ulcer
}

\author{
Thomas Junghanss
}

For decades surgical excision has been the standard therapy for Buruli ulcer (BU) with several drawbacks, however [1]. Surgery is traumatizing with extensive soft tissue excisions required to prevent relapse. Long hospitalisation is expensive for both health services and patient families. Since 2004 an antibiotic regimen consisting of rifampicin and streptomycin for 8 weeks is recommended by WHO [2, 3]. Its efficacy has been demonstrated in various studies including a randomized controlled trial [4]. The parenteral application of streptomycin over 8 weeks is a substantial disadvantage in settings with limited resources and particularly in children who are mostly affected by BU in Africa. Ototoxicity has additionally been identified as a critical and prohibitive side effect [5]. In 2017 the WHO Technical Advisory Group on $\mathrm{BU}$ decided that the $\mathrm{WHO}$ recommendation for treatment should be changed to rifampicin and oral clarithromycin pending the publication of the full results of a recently completed clinical trial. On a national level, this combination has already been introduced in various countries.

The temperature sensitivity of Mycobacterium ulcerans has long been recognized [6-9]. M. ulcerans differs from most other pathogenic mycobacteria in that it grows best at $30-33{ }^{\circ} \mathrm{C}$ and not above $37^{\circ} \mathrm{C}$ [8]. Meyers et al. treated eight patients from Zaire maintaining a temperature of approximately $40{ }^{\circ} \mathrm{C}$ in the ulcerated area for a mean duration of 68 days [8]. There was no evidence of local recurrence during follow-up periods of up to 22 months. Based on this impressive success rate, WHO guidelines listed the application of heat as a treatment option for BU [10]. However, the heat application devices initially employed were impractical in most endemic countries.

The phase change material (PCM) sodium acetate trihydrate, which is widely used in commercial pocket heat pads offers an ideal technical solution for

T. Junghanss $(\varangle)$

Section Clinical Tropical Medicine, Department of Infectious Diseases,

Heidelberg University Hospital, Heidelberg, Germany

e-mail: thomas.junghanss@urz.uni-heidelberg.de 
thermotherapy of BU. The unique feature of PCM is its thermal energy storing capacity combined with an almost constant temperature during the liquid-solid phase transition. Sodium acetate trihydrate appears ideal with its supercooling behaviour, since, once completely molten, the material will stay liquid even when the temperature falls far below its melting point. It can thus be stored at room temperature without energy loss. With a starter, e.g. a piece of copper wire protected by a rubber tube placed within the PCM filled bag, the crystallization process is initiated when required. Once initiated, heat at constant temperature is emitted for around $6 \mathrm{~h}$. Patients can be treated overnight. Further, the melting temperature of $58{ }^{\circ} \mathrm{C}$ allows sterile gauze to be placed between the wound and the PCM bag to protect the wound and to still maintain temperatures of above $39{ }^{\circ} \mathrm{C}$ at the skin surface without the risk of burning (Fig. 1).

In summary, sodium acetate trihydrate heat packs:

- Are free from significant side effects

- Are easy to apply, rechargeable in boiling water and can be used many times

- Are non-toxic and non-hazardous to the environment

- Are sterilized between rounds of application during the recharging process

- Exert gentle pressure and thus reduce peri-lesional edema

- Protect wounds from trauma

- Urge patients and medical staff to pay attention to the wound when reapplied daily

The heat application device was tested in healthy volunteers and a mathematical model was developed and validated to predict its thermal behaviour. The thermal model allowed the prediction of skin surface temperatures and an optimization of the amount of PCM with respect to discharge time [11].

In a next step commercially available heat packs were tested in a prospective observational single centre proof-of-principle trial in Ayos/Cameroon. "Six laboratory

Fig. 1 Heat packs, sterile gauze and elastic bandage are the ingredients needed for thermotherapy

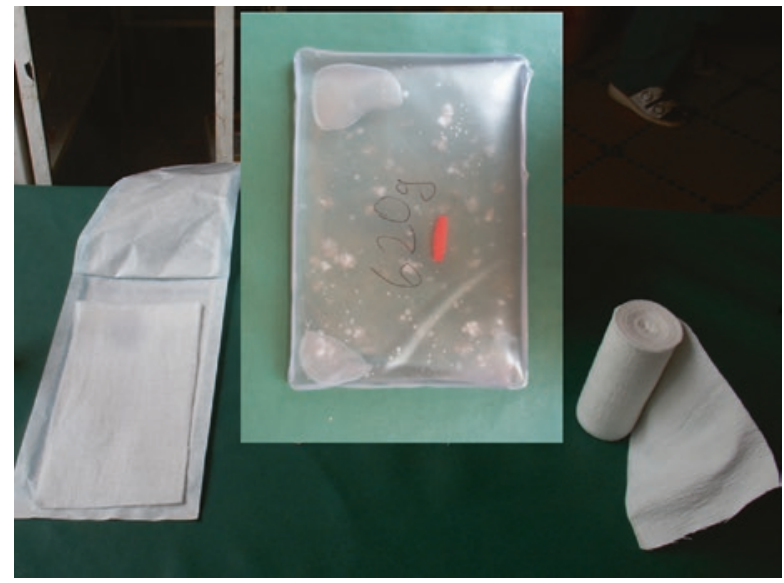


reconfirmed patients with ulcerative BU lesions received 28-31 (ulcers $<2 \mathrm{~cm}$ ) or 50-55 (ulcers $>2 \mathrm{~cm}$ ) days of thermotherapy with the PCM sodium acetate trihydrate as heat application system. All patients enrolled in the trial completed the heat treatment. Being completely mobile during the well-tolerated heat application, acceptability of the PCM bandages was very high. In patients with smaller ulcers, wounds healed completely without further intervention. Patients with large defects had skin grafting after successful heat treatment. Heat treatment was not associated with marked increases in local inflammation or the development of ectopic lymphoid tissue. One and a half years after completion of treatment, all patients were relapse-free" [12].

To confirm the findings of the pilot study a phase II open label single centre noncomparative clinical trial (ISRCTN 72102977) under GCP standards was carried out in Cameroon.

Laboratory confirmed BU patients received up to 8 weeks of heat treatment. The efficacy was assessed based on the endpoints 'absence of clinical BU specific features' or 'wound closure' within 6 months ("primary cure"), and 'absence of clinical recurrence within 24 month' ("definite cure"). Of 53 enrolled patients, 51 $(96 \%)$ had ulcerative disease. Sixty two percent were classified as World Health Organization category II, 19\% each as category I and III. The average lesion size was $45 \mathrm{~cm}^{2}$. Within 6 months after completion of heat treatment, 92.4\% (49 of 53, 95\% confidence interval (CI), 81.8-98.0\%) achieved cure of their primary lesion. At 24 months follow-up $83.7 \%$ (41 of 49, 95\% CI, 70.3-92.7\%) of patients with primary cure remained free of recurrence (see Fig. 2). Heat treatment was well tolerated and the only adverse effects were occasional mild local skin reactions [13].

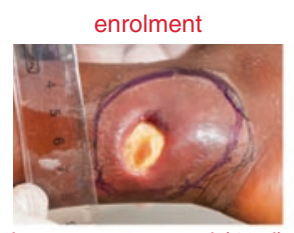

heat treatment end $(40 \mathrm{~d})$

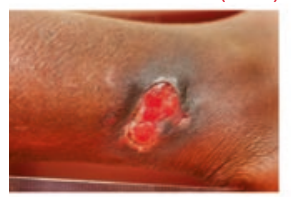

3 month FU

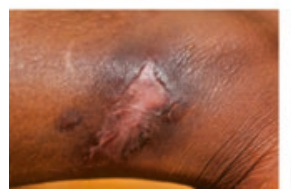

week 1

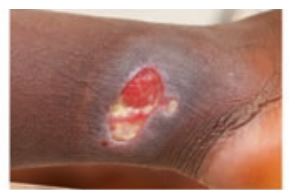

week 8

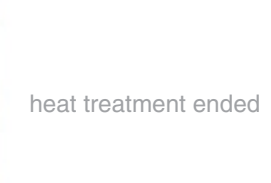

6 month FU

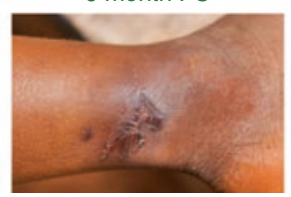

week 2

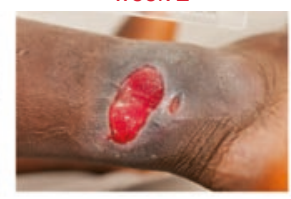

1 month FU

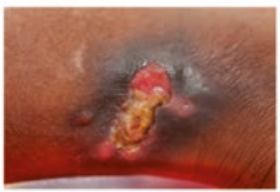

12 month FU

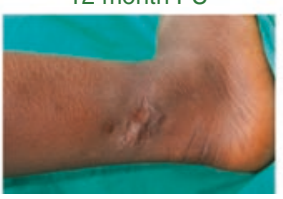

week 4

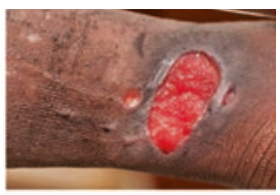

2 month FU

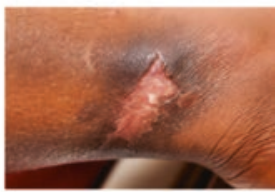

24 month FU (+4 days)

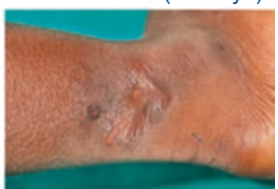

Fig. 2 Clinical evolution of patient 14 (laboratory confirmed ulcer of the right lateral ankle) exemplary for all patients. Images of all other patients are available as Supplemental Figures 1-65 of [13]. Pictures were taken within a range of \pm 2 days (heat treatment period), \pm 15 days (follow-up (FU) months $1-3$ ), \pm 30 days (FU month 6 ) and \pm 60 days (FU months $12+24$ months) from the designated time points (Source: [13]) 
Interestingly, there is evidence from other studies and our own observations that after controlling the bulk of the mycobacterial burden remaining viable $M$. ulcerans may be eliminated by the immune system without additional specific treatment. Equally, confirmed BU nodules distant to heat treated primary lesions healed (see Fig. 3). Protective immune responses may be triggered by thermotherapy and chemotherapy $[4,14,15]$ but not surgery, where the mycobacterial antigens are largely removed [13].

Importantly, the skin lesions of ten patients, for which laboratory testing for $M$. ulcerans was negative, also healed under thermotherapy, indicating that heat might have a positive effect on the healing of wounds in general. This is encouraging for regions with limited access to laboratory confirmation and where quality assurance, in particular of PCR in reference labs, remains a problem [13]. With respect to reliability of PCR-based laboratory diagnosis it has to be noted that in a multicentre
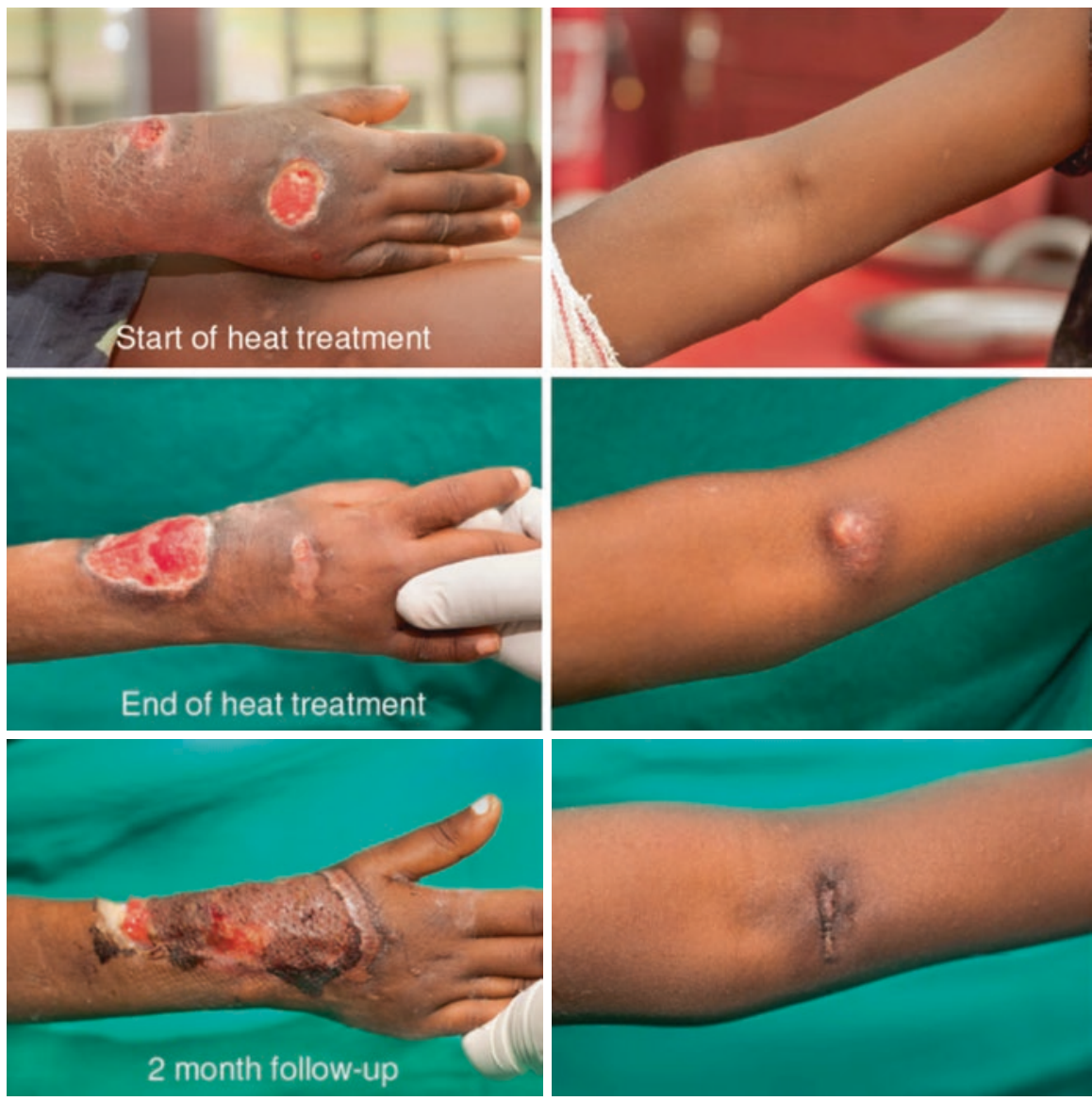

Fig. 3 Distant healing in a patient with a BU lesion at the right wrist and a laboratory confirmed BU nodule at the left elbow. Both of the lesions healed after heat was applied to the right wrist only (Source: [13]) 
external quality assessment for PCR detection of $M$. ulcerans only around one third of the participating laboratories had more than $90 \%$ concordant results [16].

Both, the proof-of-principle and the phase II trial showed that thermotherapy is well tolerated and effective. Daytime activities including schooling are not interrupted. In addition to its specific effect, heat treatment appears to promote wound healing by increasing blood circulation, reducing edema through gentle compression and protecting the wound. Renewal of heat packs is inevitably connected to inspection and care for the wound.

The thermotherapy-specific cost of heat packs and recharging for $10 \mathrm{~min}$ in boiling water are small. Nursing time mainly goes into wound management, which is not different from chemotherapy patients. Positioning of heat packs is straightforward and nurses are confident in heat pack application after few demonstrations.

In communities with limited resources treatment modalities which can be applied in early undifferentiated stages of diseases without causing harm and with the benefit of preventing advancement into severe complicated disease make a difference to peoples' lives. All wounds "count" and the patient's priority "I want to have my skin closed" (independent of the cause) is respected and promoted. This follows the broad understanding of universal health coverage [17]. The population impact lies in the rapid closure of a substantial proportion of wounds at the community level [18]. This avoids life threatening sequelae and disability and prevents the development of chronic wounds and the need for advanced treatment at the secondary or tertiary health services level. It saves costs both for the patient and the health care system. Patients with wounds prone to secondary bacterial infection benefit from treatment in the periphery because they are not exposed to nosocomial infections which are more frequent at higher levels of health services. This, again, saves people from sufferance and costs.

In summary, based on the results of the two studies with PCM heat packs, "thermotherapy can be considered an alternative to chemotherapy as primary treatment for BU for several reasons: it is highly effective, is easy to apply, cheap, well tolerated, free of relevant adverse effects, has nonspecific positive effects on wound healing and does not compromise wound healing in non-BU lesions in cases of misclassification. This is an undisputable advantage in settings where treatment decisions need to rely primarily on clinical diagnosis. Changing of heat packs urges health staff and patients to take notice of the wound and thus increases the probability of regular wound care. Heat therapy has potential as home remedy for BU lesions suspected to be BU, ideally, combined with general wound management" [13].

\section{References}

1. Junghanss T, Johnson RC, Pluschke G (2014) Mycobacterium ulcerans disease. In: Farrar J et al (eds) Manson's tropical diaseses. Elsevier Saunders, Philadelphia, pp 519-531

2. WHO, Asiedu K (2004) Provisional guidance on the role of specific antiobiotics in the management of Mycobacterium ulcerans disease (Buruli ulcer), vol 39. WHO/Department of Communicable Disease Prevention, Control and Eradication, Geneva

3. WHO, Asiedu K (2012) Treatment of Mycobacterium ulcerans disease (Buruli Ulcer). WHO/ Department of Control of Neglected Tropical Diseases, Geneva, p 73 
4. Nienhuis WA et al (2010) Antimicrobial treatment for early, limited Mycobacterium ulcerans infection: a randomised controlled trial. Lancet 375(9715):664-672

5. Klis S et al (2014) Long term streptomycin toxicity in the treatment of Buruli ulcer: follow-up of participants in the BURULICO drug trial. PLoS Negl Trop Dis 8(3):e2739

6. Glynn PJ (1972) The use of surgery and local temperature elevation in mycobacterium ulcerans infection. Aust N Z J Surg 41(4):312-317

7. Meleney FL, Johnson BA (1950) Supplementary report on the case of chronic ulceration of the foot due to a new pathogenic mycobacterium (MacCallum). Ann Soc Belg Med Trop (1920) 30(6):1499-1503

8. Meyers WM, Shelly WM, Connor DH (1974) Heat treatment of Mycobacterium ulcerans infections without surgical excision. Am J Trop Med Hyg 23(5):924-929

9. Reid IS (1967) Mycobacterium ulcerans infection: a report of 13 cases at the Port Moresby General Hospital, Papua. Med J Aust 1(9):427-431

10. WHO, Asiedu K, Scherpbier R, Raviglione M (2000) Buruli ulcer: Mycobacterium ulcerans infection. WHO/Department of Disease Control, Prevention and Eradication, Geneva, p 160

11. Braxmeier $\mathrm{S}$ et al (2009) Phase change material for thermotherapy of Buruli ulcer: modelling as an aid to implementation. J Med Eng Technol 33(7):559-566

12. Junghanss T et al (2009) Phase change material for thermotherapy of Buruli ulcer: a prospective observational single centre proof-of-principle trial. PLoS Negl Trop Dis 3(2):e380

13. Vogel M et al (2016) Local heat application for the treatment of Buruli ulcer: results of a phase II open label single center non comparative clinical trial. Clin Infect Dis 62(3):342-350

14. Beissner M et al (2012) Spontaneous clearance of a secondary Buruli ulcer lesion emerging ten months after completion of chemotherapy--a case report from Togo. PLoS Negl Trop Dis 6(7): 1747

15. Ruf MT et al (2011) Secondary Buruli ulcer skin lesions emerging several months after completion of chemotherapy: paradoxical reaction or evidence for immune protection? PLoS Negl Trop Dis 5(8):e1252

16. Eddyani M et al (2014) Multicenter external quality assessment program for PCR detection of Mycobacterium ulcerans in clinical and environmental specimens. PLoS One 9(2):e89407

17. Sakolsatayadorn P, Chan M (2017) Breaking down the barriers to universal health coverage. Bull World Health Organ 95(2):86

18. Addison NO et al (2017) Assessing and managing wounds of Buruli ulcer patients at the primary and secondary health care levels in Ghana. PLoS Negl Trop Dis 11(2):e0005331

Open Access This chapter is licensed under the terms of the Creative Commons Attribution 4.0 International License (http://creativecommons.org/licenses/by/4.0/), which permits use, sharing, adaptation, distribution and reproduction in any medium or format, as long as you give appropriate credit to the original author(s) and the source, provide a link to the Creative Commons license and indicate if changes were made.

The images or other third party material in this chapter are included in the chapter's Creative Commons license, unless indicated otherwise in a credit line to the material. If material is not included in the chapter's Creative Commons license and your intended use is not permitted by statutory regulation or exceeds the permitted use, you will need to obtain permission directly from the copyright holder.

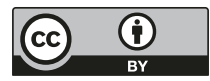

